Pacific Journal of Mathematics

ORDERS IN SIMPLE ARTINIAN RINGS ARE STRONGLY 


\section{ORDERS IN SIMPLE ARTINIAN RINGS ARE STRONGLY EQUIVALENT TO MATRIX RINGS}

\section{JULIUS ZELMANOWITZ}

The result indicated by the title will be proved. More specifically stated: when $R$ is a left order in a simple artinian ring $Q$, there exist matrix units $\left\{e_{i j}\right\}$ for $Q$ and an element $r \in D$, where $D$ is the intersection of the centralizer of $\left\{e_{i j}\right\}$ with $R$, such that $r R r \cong \sum D e_{i j}$ and $\sum r D e_{i j} \leqq R$. The Faith-Utumi theorem is an immediate consequence of this relationship. Furthermore, if $R$ is either a maximal order, or is subdirectly irreducible, or is hereditary, then there is a left order $C$ in the centralizer of $\left\{e_{i j}\right\}$ which inherits the corresponding property of $R$ and such that $R$ is equivalent to the matrix ring $\sum C e_{i j}$.

Introduction. A subring $R$ of a simple artinian ring $Q$ is a left order in $Q$ if every element of $Q$ is of the form $r^{-1} s$ for some $r, s \in R$. An order in $Q$ is a right and left order. Two left orders $R$ and $R^{\prime}$ in $Q$ are equivalent if there exist units $p, q, p^{\prime}, q^{\prime}$ of $Q$ with $p R q \subseteq R^{\prime}$ and $p^{\prime} R^{\prime} q^{\prime} \leqq R$; one then writes $R \sim R^{\prime}$. A maximal left order in $Q$ is a left order in $Q$ which is maximal in its equivalence class. It is assumed throughout that all left orders are inside a fixed simple artinian ring $Q$, and also that rings do not contain identity elements unless specifically indicated.

In the classical situation, by which is meant the theory of maximal orders over a Dedekind domain [2], all the maximal orders are equivalent. This remains true in the more general situation of Dedekind orders [9], and there exists in each equivalence class a matrix ring over a (not necessarily commutative) integral domain.

The first main result of this paper in $\S 2$ shows that given a (left) order $R$ in $Q$ there exist matrix units $\left\{e_{i j}\right\}$ for $Q$ with centralizer $\Delta$ and an element $r \in D=\Delta \cap R$ with $r R r \leqq \sum D e_{i j}, r \sum D e_{i j} \leqq R$, and $\sum D e_{i j} r \leqq R$; as expected, $D$ is a (left) order in $\Delta$. Thus, in particular, $R$ contains the matrix order $\sum r D e_{i j}$, giving the conclusion of the Faith-Utumi theorem [4]; and $R \sim \sum D e_{i j}$ [11], with a somewhat stronger condition actually satisfied. The additional information enables one to consider the important special cases when $R$ is a maximal, or a subdirectly irreducible, or a left hereditary left order. In each of these cases, a maximal left order $C \leqq \Delta$ is chosen with the same property as $R$ and with $r \sum C e_{i j} r \leqq R$ and $r R r \leqq \sum C e_{i j}$. These are treated in $\S 3-\S 5$, where partial results are also obtained for simple orders. The method of proof involves only the machinery of 
linear algebra over Ore domains.

1. Preliminaries. The reader is assumed to be familiar with Goldie's characterization of (left) orders in simple artinian rings [5], with the definition and use of Morita contexts in this setting (cf. [1], [10]), and all attendant concepts (uniform module, essential submodule, and so on).

Throughout, $R$ will denote a fixed (left) order in a simple artinian ring $Q, M$ will be a fixed uniform left ideal of $R, N=\operatorname{Hom}_{R}(M, R)$, $E=\operatorname{End}_{R} M$; and, except where specifically indicated otherwise, attention will be directed to the standard Morita context $(R, M, N, E)$ with bimodule maps (, ): $M \otimes_{E} N \rightarrow R$ and [, ]: $N \otimes_{R} M \rightarrow E$ defined via $(m, n)=(m) n, m^{\prime}[n, m]=\left(m^{\prime}, n\right) m$ for all $m, m^{\prime} \in M, n \in N$ (homomorphisms being written opposite scalars). Observe that (, ) and [, ] are nonsingular in all four variables. The well-known results presented in this section are of fundamental importance in the sequel.

Lemma 1.1. $E=\operatorname{End}_{R} M$ is a (left) order in the division ring $\operatorname{End}_{Q} Q M$.

Proof. $Q M$ is a minimal left ideal of $Q$ and is the $R$-injective hull of $M$. Hence one may regard $E=\operatorname{End}_{R} M$ as a subring of the division ring $\Delta=\operatorname{End}_{Q} Q M$. Given $\varphi \in \Delta$, set $M_{0}=M \varphi^{-1} \cap M$. Then $0 \neq\left[N, M_{0} \varphi\right]=\left[N, M_{0}\right] \varphi \subseteq E \varphi \cap E$, and it follows that $E$ is a left order in $\Delta$.

Next suppose that $R$ is also a right order in $Q$. Then one may regard $\left[N, M_{0}\right]$ as a right ideal of $\operatorname{End}_{R} M_{0}$ (by restricting the action of $N$ to $\left.M_{0}\right)$. Moreover, $\operatorname{End}_{R} M_{0}$ is a right order in $\Delta$ because $M_{0} \varphi\left[N, M_{0}\right] \subseteq(M, N) M_{0} \subseteq M_{0}$. Since $\left[N, M_{0}\right]$ is also a left ideal of $E$, it follows that $E$ is a right order in $\Delta$.

Lemma 1.2. (Dual Basis Lemma) There exist elements

$$
m_{1}, m_{2}, \cdots, m_{t} \in M, n_{1}, n_{2}, \cdots, n_{t} \in N, 0 \neq a \in E, r=\sum_{i, j=1}^{t}\left(m_{i}, n_{i}\right) \in R
$$

satisfying: ${ }_{E} N$;

(i) $n_{1}, n_{2}, \cdots, n_{t}$ is a maximal linearly independent subset of

(ii) $\left[n_{i}, m_{j}\right]=\delta_{i j} a$ for $i$ and $j$ (where $\delta_{i j}$ is the Kronecker delta);

(iii) $r$ is a regular element of $R$ (i.e., $r$ is a unit in $Q$ );

(iv) $n_{i} r=a n_{i}$ and $r m_{i}=m_{i} a$ for each $i$.

Proof. $N=\operatorname{Hom}_{R}(M, R)$ can be regarded as an essential $E$-sub- 
module of $\hat{N}=\operatorname{Hom}_{Q}(Q M, Q)$, and the latter is a finite dimensional vector space over $\Delta=\operatorname{End}_{Q} Q M$. Thus $\hat{N}$ is the $E$-injective hull of $N$, and ${ }_{E} N$ is finite dimensional and torsion-free. This being the situation, proofs of the lemma may be found in [1] and [10], except for the last assertion that $r m_{i}=m_{i} a$ for each $i$. To see this, it suffices to show that $\left[n_{j}, r m_{i}\right]=\left[n_{j}, m_{i} a\right]$ for each $j$; and this is evident since $\left[n_{j}, r m_{i}-m_{i} a\right]=a\left[n_{j}, m_{i}\right]-\left[n_{j}, m_{i}\right] a=\delta_{i j}\left(a^{2}-a^{2}\right)=0$.

2. Main results. The notation in this section continues that of $\S 1$, and the notation now introduced will be followed consistently. All sums will be taken over the integers from 1 to $t$.

Observe that

$$
r\left(m_{i}, n_{j}\right)=\sum_{k}\left(m_{k}, n_{k}\right)\left(m_{i}, n_{j}\right)=\sum_{k}\left(m_{k}\left[n_{k}, m_{i}\right], n_{j}\right)=\left(m_{i} a, n_{j}\right) .
$$

Similarly, $\left(m_{i} n_{j}\right) r=\left(m_{i}, a n_{j}\right)$, so that

$$
r\left(m_{i}, n_{j}\right)=\left(m_{i}, n_{j}\right) r \text { for all } 1 \leqq i, j \leqq t .
$$

Thus defining

$$
e_{i j}=r^{-1}\left(m_{i}, n_{j}\right)=\left(m_{i}, n_{j}\right) r^{-1},
$$

it is easy to check that $\left\{e_{i j}: 1 \leqq i, j \leqq t\right\}$ is a set of matrix units for Q. Set

$$
\Delta=\left\{q \in Q: q e_{i j}=e_{i j} q \text { for all } 1 \leqq i, j \leqq t\right\},
$$

and let $D=\Delta \cap R$.

Clearly then $\Delta$ is a division ring and $Q=\sum_{i, j} \Delta e_{i j} \cong \Delta_{t}$.

Let $R_{0}=\left\{\sum_{i, j}\left(m_{i} b_{i j}, n_{j}\right): b_{i j} \in E\right\}, D_{0}=\left\{\sum_{i}\left(m_{i} b, n_{i}\right): b \in E\right\}$. Both $R_{0}$ and $D_{0}$ are subrings of $R$. They are related as follows.

\section{Lemma 2.1. $D_{0} \subseteq D$ and $R_{0}=\sum_{i, j} D_{0} e_{i j}$.}

Proof. Let $\sum_{i}\left(m_{i} b, n_{i}\right) \in D_{0}, b \in E$. Then for any choice of $k$ and $h$,

$$
\sum_{i}\left(m_{i} b, n_{i}\right) e_{k h}=\sum_{i}\left(m_{i} b, n_{i}\right)\left(m_{k}, n_{h}\right) r^{-1}=\left(m_{k} b, a n_{h}\right) r^{-1}=\left(m_{k} b, n_{h}\right) ;
$$

and similarly, $e_{k h} \sum_{i}\left(m_{i} b, n_{i}\right)=\left(m_{k} b, n_{h}\right)$. Hence $D_{0} \subseteq D$.

Now given $\sum_{i, j}\left(m_{i} b_{i j}, n_{j}\right) \in R_{0}, b_{i j} \in E$; for each $1 \leqq i, j \leqq t$, set $r_{i j}=\sum_{k}\left(m_{k} b_{i j}, n_{k}\right) \in D_{0}$. Then

$$
r_{i j} e_{i j}=\sum_{k}\left(m_{k} b_{i j}, n_{k}\right)\left(m_{i}, n_{j}\right) r^{-1}=\left(m_{i} b_{i j}, a n_{j}\right) r^{-1}=\left(m_{i} b_{i j}, n_{j}\right) .
$$

Thus $R_{0}=\sum_{i, j} D_{0} e_{i j}$.

TheOREm 2.2. Let $R$ be a (left) order in $Q$. Then 
(i) $r \in D, \sum_{i, j} r D e_{i j} \leqq R$, and $\sum_{i, j} D e_{i j} r \leqq R$.

(ii) $r R r \subseteq R_{0} \subseteq \sum_{i, j} D e_{i j}$.

(iii) $R \sim R_{0} \sim \sum_{i, j} D e_{i j}$.

(iv) $D_{0}$ and $D$ are equivalent (left) orders in $\Delta$.

Proof. That $r \in D$ is obvious from the definition of $r$ and (2). $\sum_{i, j} r D e_{i j}=\sum_{i, j} e_{i j} r D=\sum_{i, j}\left(m_{i}, n_{j}\right) D \subseteq R$, and similarly $\sum_{i, j} D e_{i j} r \subseteq R$.

$$
\begin{aligned}
r R r & =\sum_{i}\left(m_{i}, n_{i}\right) R \sum_{j}\left(m_{j}, n_{j}\right)=\sum_{i, j}\left(m_{i}\left[n_{i} R, m_{j}\right], n_{j}\right) \subseteq R_{0} \\
& =\sum_{i, j} D_{0} e_{i j} \subseteq \sum_{i, j} D e_{i j} .
\end{aligned}
$$

(iii) is a consequence of (i) and (ii). Thus in particular, $R_{0}=\sum_{i, j} D_{0} e_{i j}$ and $\sum_{i, j} D e_{i j}$ are also (left) orders in $Q$. This implies that $D_{0}$ and $D$ must be (left) orders in $\Delta$. It remains to prove that $D$ and $D_{0}$ are equivalent. While this follows from (iii), it is useful to observe that in fact

$$
r D r \subseteq D_{0}
$$

To see this it suffices to verify that

$$
\left[n_{i} d, m_{j}\right]=\delta_{i j}\left[n_{1} d, m_{1}\right] \text { for any } 1 \leqq i, j \leqq t \text { and } d \in D \text {. }
$$

Now, $r^{-1}\left(m_{i}\left[n_{i} d, m_{j}\right], n_{j}\right) r^{-1}=e_{i i} d e_{j j}=d e_{i i} e_{j j}=0$ when $i \neq j$. Hence $\left(m_{i}\left[n_{i} d, m_{j}\right], n_{j}\right)=0$, and so $a\left[n_{i} d, m_{j}\right] a=\left[n_{i}, m_{i}\right]\left[n_{i} d, m_{j}\right]\left[n_{j}, m_{j}\right]=0$, which establishes that $\left[n_{i} d, m_{j}\right]=0$ when $i \neq j$. Similarly,

$$
r^{-1}\left(m_{i}\left(\left[n_{i} d, m_{i}\right]-\left[n_{1} d, m_{1}\right]\right), n_{i}\right)=e_{i i} d e_{i i}-e_{i 1} d e_{1 i}=0,
$$

from which it follows as above that $\left[n_{i} d, m_{i}\right]-\left[n_{1} d, m_{1}\right]=0$.

Corollary 2.3. (Faith-Utumi [4]) Given a (left) order $R$ in a simple artinian ring $Q$ there exist matrix units $\left\{e_{i j}\right\}$ for $Q$, and a (left) order $C$ in the centralizer of $\left\{e_{i j}\right\}$ such that $C \leqq R$ and $\sum_{i, j} C e_{i j} \leqq R$.

Proof. $C=r D$ is a right ideal of $D$, and hence $C$ is a (left) order in $\Delta$.

3. Maximal orders. The results of the previous section facilitate a rapid treatment of maximal orders.

THEorem 3.1. If $R$ is a maximal (left) order in $Q$, then there exists a maximal (left) order $C$ in $\triangle$ such that $r R r \subseteq \sum_{i, j} C e_{i j}$ and $\sum_{i, j} r C e_{i j} r \subseteq R$.

Proof. Of course $1 \in R$, since $R$ is a maximal left order in $Q$. Let $C$ be any left order in $\Delta$ containing $D$ and equivalent to $D$. 
Then without loss of generality, it may be assumed that there exists $d, d^{\prime} \in D$ with $d C d^{\prime} \leqq D$. Consider $R^{\prime}=R+R r\left(\sum_{i, j} C e_{i j}\right) r R ; R^{\prime}$ is a left order in $Q$ because $R \subseteq R^{\prime}$ and $r R r \subseteq \sum_{i, j} D e_{i j} \subseteq \sum_{i, j} C e_{i j}$. Also $R^{\prime}$ is equivalent to $R$ because

$$
r d r R^{\prime} r d^{\prime} \subseteq r d \sum_{i, j} C e_{i j} d^{\prime} \subseteq r \sum_{i, j} D e_{i j} \subseteq R
$$

By the maximality of $R$ it must be the case that $R=R^{\prime}$. In particular $r\left(\sum_{i, j} C e_{i j}\right) r=\sum_{i, j} r C r e_{i j} \leqq R$. Thus $r C r \leqq R \cap \Delta=D$.

Hence given an arbitrary left order $C$ in $\Delta$ with $D \subseteq C$ and $D \sim C$, it is always the case that $r C r \subseteq D$. This enables one to apply Zorn's Lemma to choose a maximal such $C$. The rest of the theorem is clear.

REMARK. It would be of interest to learn whether necessarily $C=D$ in the above theorem; especially in the case where $M$ is a basic left ideal. The answer is not known to the author at this time.

4. Simple orders. The obvious question for simple orders with 1 is whether they are equivalent to matrix rings over simple Ore domains. The analogous question for Morita-equivalence is not as yet settled (see [3]). Unfortunately, even in the present simplified setting one encounters the same difficulties as arise for the Morita-equivalence problem. Recall that a ring is subdirectly irreducible if it has a unique nonzero minimal ideal. As usual the notation follows that of prior sections.

THEOREM 4.1. If $R$ is a subdirectly irreducible (left) order in $Q$, then there exists a subdirectly irreducible (left) order $C$ in $\triangle$ such that $\sum_{i, j} r C r e_{i j} \subseteq R$ and $r R r \subseteq \sum_{i, j} C e_{i j}$. Moreover, if $R$ is maximal in $Q, C$ can be chosen maximal in $\Delta$.

Proof. When $R$ is a maximal (left) order, choose $C$ containing $D$ as in Theorem 3.1; otherwise, take $C=D$. It remains only to verify that $C$ is subdirectly irreducible. For this, let $I$ be the unique minimal ideal of $R$, and let $A$ be any nonzero ideal of $S=\sum_{i, j} C e_{i j}$. Then $\operatorname{RrArR}$ is a nonzero ideal of $R$, and so $I \subseteq R r A r R$. Hence $r I r \subseteq(r R r) A(r R r) \subseteq S A S \subseteq A$. Since $A$ was arbitrary, $r I r \neq 0$ is contained in the intersection of the ideals of $S$. Such ideals are of the form $\sum_{i, j} B e_{i j}$ for $B$ an ideal of $C$; and from this it is immediate that $C$ has a minimal ideal.

CoROLlaRY 4.2. If $R$ is a simple (left) order with 1, then there exists a subdirectly irreducible maximal (left) order $C$ in $\Delta$ such that $\sum_{i, j} r C r e_{i j} \leqq R$ and $r R r \subseteq \sum_{i, j} C e_{i j}$. 
LEMMA 4.3. $\quad r^{-1} D_{0}$ is a ring isomorphic to $E$ under the homomorphism defined via $b \rightarrow r^{-1} \sum_{i}\left(m_{i} b, n_{i}\right)$ for $b \in E$.

Proof. The verification is entirely routine once it is proved that the map is multiplicative; and for this it suffices to demonstrate that for any $b, c \in E$,

$$
\sum_{i}\left(m_{i} b, n_{i}\right) r^{-1} \sum_{j}\left(m_{j} c, n_{j}\right)=\sum_{i}\left(m_{i} b c, n_{i}\right) .
$$

To see this, choose $b_{1}, c_{1} \in E$ with $0 \neq b_{1} a b=c_{1} a^{2}$ (this is possible because $E$ is a left Ore domain), and then multiply the difference of both sides of the equation in (5) by the invertible element $\sum_{k}\left(m_{k} b_{1}, n_{k}\right)$ to obtain zero.

THEOREM 4.4. Suppose that $R$ is a simple (left) order with 1, and that $R$ has a projective uniform left ideal. Then $r^{-1} D_{0}$ is a simple (left) order with 1 in $\Delta$ and $R \sim \sum_{i, j} r^{-1} D_{0} e_{i j}$.

Proof. Choose ${ }_{R} M$ to be projective. Then by [6; Lemma 4], ${ }_{R} M$ is finitely generated, and hence is an $R$-progenerator. It follows that $E$ is simple, and then by the preceding lemma $r^{-1} D_{0}$ is simple. Now $D_{0} \subseteq r^{-1} D_{0}$, and $D_{0}$ is a (left) order by Theorem 2.2. Hence the same is true for $r^{-1} D_{0}$. Finally, $r \sum_{i, j} r^{-1} D_{0} e_{i j}=\sum_{i, j} D_{0} e_{i j}=R_{0} \leqq R$ and $R r=r^{-1}(r R r) \subseteq r^{-1} R_{0}=\sum_{i, j} r^{-1} D_{0} e_{i j}$.

REMARK. In the situation of the preceding corollary, it has been seen that $r^{-1} D_{0}$ is Morita-equivalent to $R$. Therefore, any categorical property of $R$ will be inherited by $r^{-1} D_{0}$.

5. Dedekind prime rings. A maximal (left) hereditary (left) noetherian (left) order $R$ in $Q$ is called a (left) Dedekind prime ring. All orders in this section are assumed to contain the identity element.

THEOREM 5.1. If $R$ is a left hereditary (left) order in $Q$, then $r^{-1} D_{0}$ is a (left) hereditary (left) order in $\Delta$, and $R \sim \sum_{i, j} r^{-1} D_{0} e_{i j}$.

Proof. Since a (left) hereditary left order is left noetherian by [8; Theorem 3.11], $E=\operatorname{End}_{R} M$ is the endomorphism ring of a finitely generated projective module over a (left) hereditary ring. By [9; Lemma 4.4], $E$ is (left) hereditary, and then Lemma 4.3 ensures that this is true for $r^{-1} D_{0}$.

Corollary 5.2. Suppose that $R$ is a (left) Dedekind prime ring. Then $r^{-1} D_{0}$ is a (left) Dedekind prime domain, and $R \sim \sum_{i, j} r^{-1} D_{0} e_{i j}$. 
Proof. It remains only to observe that $E=\operatorname{End}_{R} M$ is a maximal (left) order in $\Delta$. This can be found in [7; Lemma 1.7].

\section{REFERENCES}

1. S. A. Amitsur, Rings of quotients and Morita contexts, J. Algebra, 17 (1971), 273298.

2. M. Auslander and O. Goldman, Maximal orders, Trans. Amer. Math. Soc., 97 (1960), $1-24$.

3. C. Faith, $A$ correspondence thoerem for projective modules and the structure of simple noetherian rings, Bull. Amer. Math. Soc., 77 (1971), 338-342.

4. C. Faith and Y. Utumi, On noetherian prime rings, Trans. Amer. Math. Soc., 114 (1965), 53-60.

5. A. W. Goldie, The structure of prime rings under ascending chain condition, Proc. London Math. Soc., (3) 8 (1958), 589-608.

6. R. Hart, Simple rings with uniform right ideals, J. London Math. Soc., 42 (1967), 614-617.

7. R. Hart and J. C. Robson, Simple rings and rings Morita equivalent to Ore domains, Proc. London Math. Soc., (3) 21 (1970), 232-242.

8. L. Levy, Torsion-free and divisible modules over non-integral domains, Canada J. Math., 15 (1963), 132-151.

9. J. C. Robson, Non-commutative Dedekind rings, J. Algebra, 9 (1968), 249-265.

10. J. Zelmanowitz, Semiprime modules with maximum conditions, J. Algebra, 25 (1973), 554-574.

11. C. Faith, Orders in simple artinian rings, Trans. Amer. Math. Soc., 114 (1965), 61-64.

Received July 31, 1972. This research was supported in part by National Science Foundation grant GP-34098.

University of California, Santa Barbara 



\section{PACIFIC JOURNAL OF MATHEMATICS}

\section{EDITORS}

RICHARD ARENS (Managing Editor)

University of California

Los Angeles, California 90024

\author{
R. A. Beaumont \\ University of Washington \\ Seattle, Washington 98105
}

J. Dugundu*

Department of Mathematics

University of Southern California

Los Angeles, California 90007

D. Gilbarg and J. Milgram

Stanford University

Stanford, California 94305

\section{ASSOCIATE EDITORS}
E. F. BECKENBACH
B. H. NeumanN
F. WOLF
K. YosHIDA

\section{SUPPORTING INSTITUTIONS}

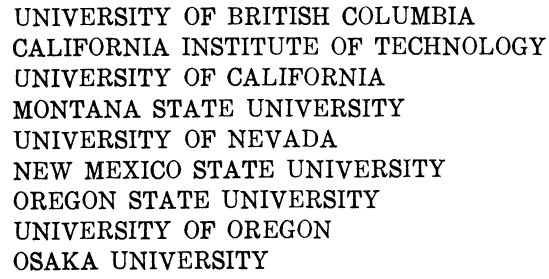

UNIVERSITY OF BRITISH COLUMBIA CALIFORNIA INSTITUTE OF TECHNOLOGY

UNIVERSITY OF CALIFORNIA

MONTANA STATE UNIVERSITY

UNIVERSITY OF NEVADA

NEW MEXICO STATE UNIVERSITY

OREGON STATE UNIVERSITY

UNIVERSITY OF OREGON

OSAKA UNIVERSITY

\author{
UNIVERSITY OF SOUTHERN CALIFORNIA \\ STANFORD UNIVERSITY \\ UNIVERSITY OF TOKYO \\ UNIVERSITY OF UTAH \\ WASHINGTON STATE UNIVERSITY \\ UNIVERSITY OF WASHINGTON \\ * * * \\ AMERICAN MATHEMATICAL SOCIETY \\ NAVAL WEAPONS CENTER
}

The Supporting Institutions listed above contribute to the cost of publication of this Journal, but they are not owners or publishers and have no responsibility for its content or policies.

Mathematical papers intended for publication in the Pacific Journal of Mathematics should be in typed form or offset-reproduced, (not dittoed), double spaced with large margins. Underline Greek letters in red, German in green, and script in blue. The first paragraph or two must be capable of being used separately as a synopsis of the entire paper. Items of the bibliography should not be cited there unless absolutely necessary, in which case they must be identified by author and Journal, rather than by item number. Manuscripts, in duplicate if possible, may be sent to any one of the four editors. Please classify according to the scheme of Math. Rev. Index to Vol. 39. All other communications to the editors should be addressed to the managing editor, or Elaine Barth, University of California, Los Angeles, California, 90024.

50 reprints are provided free for each article; additional copies may be obtained at cost in multiples of 50 .

The Pacific Journal of Mathematics is issued monthly as of January 1966. Regular subscription rate: $\$ 48.00$ a year (6 Vols., 12 issues). Special rate: $\$ 24.00$ a year to individual members of supporting institutions.

Subscriptions, orders for back numbers, and changes of address should be sent to Pacific Journal of Mathematics, 103 Highland Boulevard, Berkeley, California, 94708.

PUBLISHED BY PACIFIC JOURNAL OF MATHEMATICS, A NON-PROFIT CORPORATION

Printed at Kokusai Bunken Insatsusha (International Academic Printing Co., Ltd.), 270, 3-chome Totsuka-cho. Shinjuku-ku, Tokyo 160, Japan.

* C. R. DePrima California Institute of Technology, Pasadena, CA 91109, will replace J. Dugundji until August 1974.

Copyright (C) 1973 by

Pacific Journal of Mathematics

All Rights Reserved 


\section{Pacific Journal of Mathematics}

Vol. 48, No. $2 \quad$ April, 1973

Mir Maswood Ali, Content of the frustum of a simplex................

Mieczyslaw Altman, Contractors, approximate identities and factorization

in Banach algebras ................................ 323

Charles Francis Amelin, A numerical range for two linear operators ...... 335

John Robert Baxter and Rafael Van Severen Chacon, Nonlinear functionals

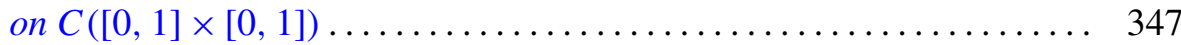

Stephen Dale Bronn, Cotorsion theories....................... 355

Peter A. Fowler, Capacity theory in Banach spaces............... 365

Jerome A. Goldstein, Groups of isometries on Orlicz spaces ........... 387

Kenneth R. Goodearl, Idealizers and nonsingular rings . ............ 395

Robert L. Griess, Jr., Automorphisms of extra special groups and

nonvanishing degree 2 cohomology ..................... 403

Paul M. Krajkiewicz, The Picard theorem for multianalytic functions . . . . 423

Peter A. McCoy, Value distribution of linear combinations of axisymmetric harmonic polynomials and their derivatives ...................

A. P. Morse and Donald Chesley Pfaff, Separative relations for

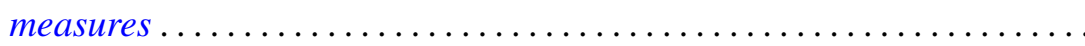

Albert David Polimeni, Groups in which $\operatorname{Aut}(G)$ is transitive on the

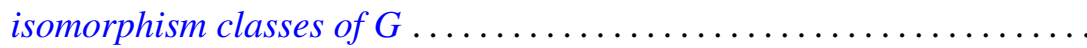

Aribindi Satyanarayan Rao, Matrix summability of a class of derived

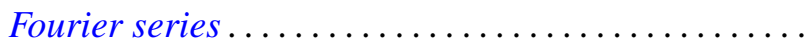

Thomas Jay Sanders, Shape groups and products

Ruth Silverman, Decomposition of plane convex sets. II. Sets associated

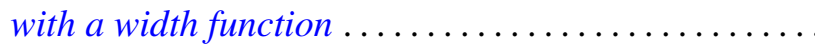

Richard Snay, Decompositions of $E^{3}$ into points and countably many flexible dendrites.............................

John Griggs Thompson, Nonsolvable finite groups all of whose local subgroups are solvable, IV ...

Robert E. Waterman, Invariant subspaces, similarity and isometric equivalence of certain commuting operators in $L_{p} \ldots$

James Chin-Sze Wong, An ergodic property of locally compact amenable

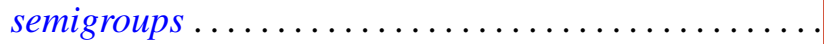

Julius Martin Zelmanowitz, Orders in simple Artinian rings are strongly equivalent to matrix rings ....................... 